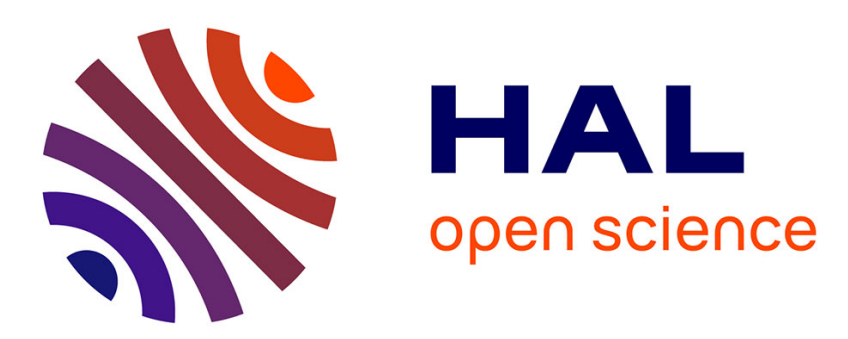

\title{
Approaches to Multivalued Mathematical Morphology Based on Uncertain Reduced Orderings
}

Mateus Sangalli, Marcos Eduardo Valle

\section{To cite this version:}

Mateus Sangalli, Marcos Eduardo Valle. Approaches to Multivalued Mathematical Morphology Based on Uncertain Reduced Orderings. Mathematical Morphology and Its Applications to Signal and Image Processing, Jul 2019, Saarbrucken, Germany. 10.1007/978-3-030-20867-7_18 . hal-02144471

\section{HAL Id: hal-02144471 \\ https://hal.science/hal-02144471}

Submitted on 30 May 2019

HAL is a multi-disciplinary open access archive for the deposit and dissemination of scientific research documents, whether they are published or not. The documents may come from teaching and research institutions in France or abroad, or from public or private research centers.
L'archive ouverte pluridisciplinaire HAL, est destinée au dépôt et à la diffusion de documents scientifiques de niveau recherche, publiés ou non, émanant des établissements d'enseignement et de recherche français ou étrangers, des laboratoires publics ou privés. 


\title{
Approaches to Multivalued Mathematical Morphology Based on Uncertain Reduced Orderings ${ }^{\star}$
}

\author{
Mateus Sangalli and Marcos Eduardo Valle \\ Department of Applied Mathematics, University of Campinas, Campinas - SP, Brazil. \\ E-mails: ra156684@ime.unicamp.br, valledime.unicamp.br
}

\begin{abstract}
Mathematical morphology (MM) is a powerful non-linear theory that can be used for signal and image processing and analysis. Although MM can be very well defined on complete lattices, which are partially ordered sets with well defined extrema operations, there is no natural ordering for multivalued images such as hyper-spectral and color images. Thus, a great deal of effort has been devoted to ordering schemes for multivalued MM. In a reduced ordering, in particular, elements are ranked according to the so-called ordering mapping. Despite successful applications, morphological operators based on reduced orderings are usually too reliant on the ordering mapping. In many practical situations, however, the ordering mapping may be subject to uncertainties such as measurement errors or the arbitrariness in the choice of the mapping. In view of this remark, in this paper we present two approaches to multivalued MM based on an uncertain reduced ordering. The new operators are formulated as the solution of an optimization problem which, apart from the uncertainty, can circumvent the false value problem and deal with irregularity issues.
\end{abstract}

Keywords: Mathematical morphology, multivalued image, optimization problem, uncertainty.

\section{Introduction}

Mathematical morphology (MM) is a powerful non-linear theory that uses geometric and topological concepts for signal and image processing and analysis [12,18]. Applications of MM include, for instance, boundary detection, image segmentation and reconstruction, pattern recognition, and signal and image decomposition $[4,8,16]$.

Apart from the geometrical interpretation inherent to many morphological operators, they can be very well defined on an algebraic structure called complete lattices $[12,14]$. A complete lattice $\mathbb{L}$ is a partially ordered non-empty set in which any subset admits both a supremum and an infimum $[3,10]$. Since the only requirement is a partial order with well-defined extreme operations, complete lattices allowed for the development of morphological operators to multivalued data, including vector-valued images such as color and hyper-spectral images $[2,13]$. In contrast to real-valued approaches, however, there is no natural ordering for vectors. Therefore, much research on vectorvalued MM has been dedicated to finding an appropriate ordering scheme for a given

\footnotetext{
* This work was supported in part by CNPq grant no. 310118/2017-4 and FAPESP grant no. 2019/02278-2.
} 
multivalued image processing task. The interested reader can find detailed discussions of multivalued MM in [1,2].

One simple approach to multivalued MM is obtained by applying gray-scale morphological operators in a component-wise manner. From the complete lattice point of view, the component-wise approach is based on the marginal ordering, also known as the product ordering. The marginal ordering is an example of a partial ordering which is not total [11]. A morphological operator based on a non-total ordering scheme may yield false values, also called false colors. A false value is an element from the set of values that does not belong to the original image and it can be a problem in some vectorvalued image processing tasks [7,17]. Furthermore, the information between bands of a vector-valued image (or channels in a color image) are ignored in a component-wise approach.

In order to circumvent the false values problem, a great deal of effort has been dedicated to multivalued morphological operators based on a total ordering scheme. Among the total ordering approaches, those obtained by combining a reduced ordering with a look-up table are particularly interesting and computationally cheap $[9,21]$. The idea behind an approach based on a reduced ordering can be summarized as follows. First, a vector-valued image is transformed into a gray-scale (usually real-valued) image using a surjective mapping $h$ called ordering mapping. Then, a flat gray-scale morphological operator is applied on the resulting image and a semi-inverse of $h$ is used to recover a vector-valued image [9]. The semi-inverse mapping can be determined using a look-up table in which the vector-values are ranked accordingly to their $h$-values [21].

In many practical situations, the $h$ mapping has a continuous range and the probability of two different vectors yield the same $h$-value is very small. In these situations, the resulting reduced ordering becomes a total ordering when restricted to the range of a vector-valued image [6]. Although total orderings avoid the appearance of false values, they are usually irregular in a metric space [6]. Specifically, Chevallier and Angulo showed that under mild conditions there always exist vectors $\mathbf{x}, \mathbf{y}, \mathbf{z}$ such that $\mathbf{x} \leq \mathbf{y} \leq \mathbf{z}$ but $d(\mathbf{x}, \mathbf{z})<d(\mathbf{x}, \mathbf{y})$, where $d$ denotes a metric and " $\leq$ " is a total order. In other words, $\mathbf{z}$ is more similar (or it is closer) to $\mathbf{x}$ than $\mathbf{y}$ in spite of the inequalities $\mathbf{x} \leq \mathbf{y} \leq \mathbf{z}$. Like false values, the irregularity issue may be a problem in some vector-valued image processing tasks.

In this paper, we propose two approaches to multivalued MM that prevent the apparition of false values but may not be defined using a total ordering and, thus, possibly avoiding the irregularity issue. In fact, the novel approaches can deal with the irregularity issue whenever it can be properly measured. The motivation behind our approaches stems from the fact that reduced orderings are also usually too reliant on the mapping $h$. The mapping $h$, however, can be subject to uncertainties such as the unavoidable measurement errors under real physical conditions when acquiring a vector-valued image. Broadly speaking, our approaches are derived by relaxing a reduced ordering but including a kind of regularization goal.

The paper is organized as follows: The next section reviews multivalued MM while Section 3 briefly presents some approaches based on reduced orderings. We address the uncertainties involved in a reduced ordering and present our approaches in Section 4. The paper finishes with some concluding remarks on Section 5. 


\section{Mathematical Morphology for Multivalued Images}

First of all, an image is a mapping from a point set $\mathcal{D}$ to a value set $\mathbb{V}$. In particular, a gray-scale image is obtained by considering $\mathbb{V} \subset \overline{\mathbb{R}}$, where $\overline{\mathbb{R}}=\mathbb{R} \cup\{+\infty,-\infty\}$. We speak of a multivalued image when $\mathbb{V} \subset \overline{\mathbb{R}}^{k}$ for $k \geq 2$. For simplicity, in this paper we shall assume that the domain $\mathcal{D}$ is a finite subset of either $E=\mathbb{R}^{2}$ or $E=\mathbb{Z}^{2}$. We denote $^{1}$ the set of all images from a domain $\mathcal{D}$ to a value set $\mathbb{V}$ by $\mathcal{V}=\mathbb{V}^{\mathcal{D}}$.

Briefly, morphological operators examine an image by probing it with a small pattern called structuring element $[12,18]$. The structuring element is used to extract useful information about the geometrical and topological structures on an image. Such as the domain of an image, we assume that a structuring element $S$ corresponds to a finite subset of either $E=\mathbb{R}^{2}$ or $E=\mathbb{Z}^{2}$ with $\operatorname{Card}(S) \ll \operatorname{Card}(\mathcal{D})$.

As pointed out in the introduction, complete lattices constitute an appropriate framework for a general theory of MM $[12,14]$. A partially ordered set $(\mathcal{L}, \leq)$ is a complete lattice if any subset $X \subset \mathcal{L}$ admits a supremum and an infimum, denoted respectively by $\bigvee X$ and $\bigwedge X$. On a complete lattice, the fundamental operators of MM, called erosion and dilation, are defined as follows using an adjunction relationship [12]:

Definition 1. Let $\mathcal{L}$ be a complete lattice. We say that $\varepsilon: \mathcal{L} \rightarrow \mathcal{L}$ and $\delta: \mathcal{L} \rightarrow \mathcal{L}$ form an adjunction if

$$
\mathbf{J} \leq \varepsilon(\mathbf{I}) \Longleftrightarrow \delta(\mathbf{J}) \leq \mathbf{I}, \quad \text { for } \mathbf{I}, \mathbf{J} \in \mathcal{L}
$$

If $\varepsilon$ and $\delta$ form an adjunction, then $\varepsilon$ is an erosion and $\delta$ is a dilation.

Erosions and dilations are the two elementary operations of MM [18]. Many other morphological operators are obtained by combining erosions and dilations. For example, their compositions yield the so-called opening and closing, which have interesting topological properties and are used as non-linear image filters [18].

Let us assume that the value set $\mathbb{V}$, equipped with a certain partial ordering " $\leq$ ", is a complete lattice. The set $\mathcal{L}=\mathbb{V}^{\mathcal{D}}$ of all images from $\mathcal{D} \subset E$ to a value set $\mathbb{V}$ is also a complete lattice with the pointwise ordering defined by $\mathbf{I} \leq \mathbf{J}$ if and only if $\mathbf{I}(p) \leq \mathbf{J}(p)$ for all $p \in \mathcal{D}$. Furthermore, given a structuring element $S \subset E$, define the operators $\varepsilon_{S}: \mathcal{L} \rightarrow \mathcal{L}$ and $\delta_{S}: \mathcal{L} \rightarrow \mathcal{L}$ as follows:

$$
\varepsilon_{S}(\mathbf{I})(p)=\bigwedge\{\mathbf{I}(p+s): s \in S, p+s \in \mathcal{D}\}, \quad \forall p \in \mathcal{D}
$$

and

$$
\delta_{S}(\mathbf{I})(p)=\bigvee\{\mathbf{I}(p+s): s \in S, p+s \in \mathcal{D}\}, \quad \forall p \in \mathcal{D} .
$$

It is not hard to show that $\varepsilon_{S}$ and $\delta_{S^{*}}$, where $S^{*}=\{-s \mid s \in S\}$ is the reflected structuring element, form an adjunction on $\mathcal{L}=\mathbb{V}^{\mathcal{D}}$ equipped with the pointwise ordering. Thus, $\varepsilon_{S}$ and $\delta_{S}$ defined by (2) and (3) are respectively an erosion and a dilation. In fact, these two operators are the most widely used (flat) elementary operations of either gray-scale and multivalued MM.

\footnotetext{
${ }^{1}$ Throughout this paper, blackboard bold capital letters such as $\mathbb{V}$ and $\mathbb{L}$ are used to denote value sets while calligraphic capital letters like $\mathcal{V}$ and $\mathcal{L}$ are used to denote sets of images.
} 


\section{Reduced Orderings}

In a reduced ordering (R-ordering) or $h$-ordering, the elements are ranked according to a surjective mapping $h: \mathcal{V} \rightarrow \mathcal{L}$, where $\mathcal{L}$ is a complete lattice [9]. Given a surjective mapping $h: \mathcal{V} \rightarrow \mathcal{L}$, referred to as the ordering mapping, the $h$-ordering is defined by

$$
\mathbf{x} \leq_{h} \mathbf{y} \Longleftrightarrow h(\mathbf{x}) \leq h(\mathbf{y}), \quad \forall \mathbf{x}, \mathbf{y} \in \mathcal{V}
$$

Although being reflexive and transitive, the binary relation " $\leq_{h}$ " may fail to be antisymmetric. Thus, an $h$-ordering " $\leq_{h}$ " is in fact a pre-order. Nevertheless, an $h$-ordering can be used to define elementary morphological operators as follows [9]:

Definition 2. Consider a surjective mapping $h$ from a value set $\mathcal{V}$ to a complete lattice $\mathcal{L}$. We say that two operators $\varepsilon^{h}, \delta^{h}: \mathcal{V} \rightarrow \mathcal{V}$ form an h-adjunction if

$$
\mathbf{y} \leq_{h} \varepsilon^{h}(\mathbf{x}) \Longleftrightarrow \delta^{h}(\mathbf{y}) \leq_{h} \mathbf{x}, \quad \text { for } \mathbf{x}, \mathbf{y} \in \mathcal{V}
$$

If $\varepsilon^{h}$ and $\delta^{h}$ form an $h$-adjunction, we say that $\varepsilon^{h}$ is an $h$-erosion and $\delta^{h}$ is an $h$ dilation.

In practice, we obtain an $h$-adjunction (an $h$-erosion and an $h$-dilation) from an adjunction (an erosion and a dilation) on $\mathcal{L}$ [9]. Precisely, if $\varepsilon^{h}: \mathcal{V} \rightarrow \mathcal{V}$ and $\delta^{h}: \mathcal{V} \rightarrow$ $\mathcal{V}$ form an $h$-adjunction, then there exist adjoint operators $\varepsilon: \mathcal{L} \rightarrow \mathcal{L}$ and $\delta: \mathcal{L} \rightarrow \mathcal{L}$ such that

$$
h \varepsilon^{h}=\varepsilon h \quad \text { and } \quad h \delta^{h}=\delta h .
$$

In other words, the evaluation of the $h$-erosion $\varepsilon^{h}$ (or the $h$-dilation $\delta^{h}$ ) by $h$ equals the erosion $\varepsilon$ (or the dilation $\delta$ ) of the evaluation by $h$. In particular, if we consider $\mathcal{L}=\bar{R}$, then the classical gray-scale elementary operators $\varepsilon$ and $\delta$ given respectively by (2) and (3) can be used to determine $h$-morphological operators.

In analogy to the complete lattice case, $h$-dilations and $h$-erosions can be combined to yield many $h$-morphological operators. For example, if $\varepsilon^{h}$ and $\delta^{h}$ form an $h$-adjunction, then $\gamma^{h}=\varepsilon^{h} \delta^{h}$ is an $h$-opening and $\phi^{h}=\delta^{h} \varepsilon^{h}$ is an $h$-closing. Alternatively, in analogy to (6), we can construct a vector-valued operator $\psi^{h}: \mathcal{V} \rightarrow \mathcal{V}$ from a morphological operator $\psi: \mathcal{L} \rightarrow \mathcal{L}$ using Proposition 1 below, which is based on the notion of $h$-increasing operator [9].

Definition 3. We say that an operator $\psi^{h}: \mathcal{V} \rightarrow \mathcal{V}$ is $h$-increasing if $\mathbf{x} \leq_{h} \mathbf{y}$ implies $\psi^{h}(\mathbf{x}) \leq{ }_{h} \psi^{h}(\mathbf{y})$.

Proposition 1. An operator $\psi^{h}: \mathcal{V} \rightarrow \mathcal{V}$ is $h$-increasing if and only if there exists an increasing operator $\psi: \mathcal{L} \rightarrow \mathcal{L}$ such that

$$
h \psi^{h}=\psi h,
$$

In this case, we write $\psi^{h} \stackrel{h}{\rightarrow} \psi$. 


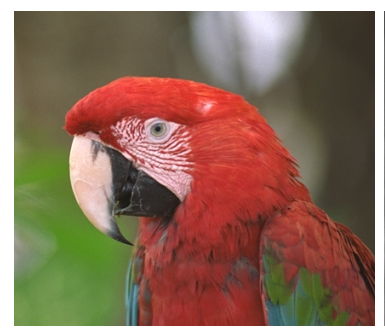

a) $\mathbf{I}$

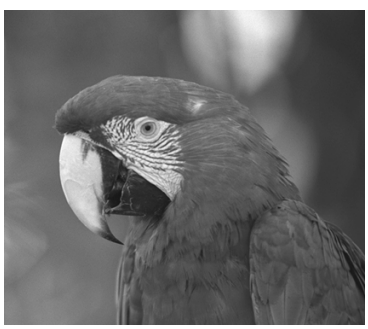

b) $h \mathbf{I}$

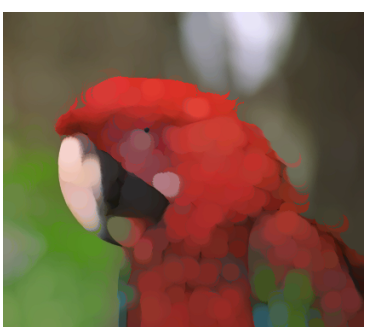

c) $\gamma_{S}^{h}(\mathbf{I})$

Fig. 1. Color image I, its evaluation $h \mathbf{I}$, and the $h$-opening $\gamma_{S}^{h}(\mathbf{I})$ by a disk of radius 10 .

From Proposition 1, any $h$-increasing operator $\psi^{h}: \mathcal{V} \rightarrow \mathcal{V}$ can be derived from an increasing operator $\psi: \mathcal{L} \rightarrow \mathcal{L}$ by means of (7). Needless to say, $h$-erosions and $h$-dilations are $h$-increasing operators. Accordingly, we have $\varepsilon^{h} \stackrel{h}{\rightarrow} \varepsilon$ and $\delta^{h} \stackrel{h}{\rightarrow} \delta$ from (6). In a similar fashion, $h$-openings and $h$-closings are $h$-increasing operators. Therefore, an $h$-opening $\gamma^{h}: \mathcal{V} \rightarrow \mathcal{V}$ and an $h$-closing $\phi^{h}: \mathcal{V} \rightarrow \mathcal{V}$ can be derived from an opening $\gamma: \mathcal{L} \rightarrow \mathcal{L}$ and a closing $\phi: \mathcal{L} \rightarrow \mathcal{L}$ such that $h \gamma^{h}=\gamma h$ and $h \phi^{h}=\phi h$.

When an $h$-morphological operator $\psi^{h}: \mathcal{V} \rightarrow \mathcal{V}$, derived from a surjective mapping $h: \mathcal{V} \rightarrow \mathcal{L}$ and an increasing operator $\psi: \mathcal{L} \rightarrow \mathcal{L}$, is applied to vector-valued images, we consider $\mathcal{V}=\mathbb{V}^{\mathcal{D}}$ and $\mathcal{L}=\mathbb{L}^{\mathcal{D}}$, where $\mathbb{V}$ denotes a vector-valued set and $\mathbb{L}$ is a complete lattice. It turns out that a mapping $h: \mathbb{V} \rightarrow \mathbb{L}$ can be extended to images $\mathbf{I} \in \mathcal{V}$ in a pointwise manner as follows:

$$
h \mathbf{I}(p)=h(\mathbf{I}(p)), \quad \forall p \in \mathcal{D} .
$$

Although we are concerned with operators for vector-valued images, we shall focus on a mapping $h: \mathbb{V} \rightarrow \mathbb{L}$ and assume it is extended to multivalued images using (8). The interested reader is referred to [21] for a review of reduced orderings in vector-valued $\mathrm{MM}$, as well as to $[1,19,5,20,15]$ for some examples of reduced orderings used in vector-valued MM that are found in the literature. Moreover, from Proposition 1, we can compute efficiently a vector-valued $h$-morphological operator $\psi^{h}$ from a classical flat gray-scale increasing operator $\psi$ using a look-up table [21]. The interested reader can find in [21] a MATLAB-style pseudo-code for computing $\psi^{h}(\mathbf{I})$ from an increasing morphological operator $\psi$ that does not introduce false values.

As far as we know, almost all reduced orderings are defined by considering mappings $h: \mathbb{V} \rightarrow \mathbb{L} \subset \overline{\mathbb{R}}$. Furthermore, we usually have $h(\mathbf{x}) \neq h(\mathbf{y})$ for distinct $\mathbf{x}$ and $\mathbf{y}$ in $\mathbf{I}(\mathcal{D})=\{\mathbf{I}(p): p \in \mathcal{D}\}$, the range of the multivalued image $\mathbf{I}$. In this case, the $h$ ordering yields a total ordering on $\mathbf{I}(\mathcal{D})$. Accordingly, although the $h$-ordering prevents the occurrence of false values (or colors), it is subject to irregularity issues [6]. A visual interpretation of this remark is illustrated in Fig. 1. Precisely, Fig. 1a) shows a color image I while Fig. 1b) depicts the gray-scale image $h \mathbf{I}$ obtained using the mapping $h: \mathbb{V}_{\mathrm{RGB}} \rightarrow[0,1]$ defined by

$$
h(\mathbf{x})=0.298936 x_{R}+0.587043 x_{G}+0.114021 x_{B}, \forall \mathbf{x}=\left(x_{R}, x_{G}, x_{B}\right) \in \mathbb{V}_{\mathrm{RGB}}
$$


where $\mathbb{V}_{\mathrm{RGB}}=[0,1] \times[0,1] \times[0,1]$. Also, Fig. 1c) shows the $h$-opening $\gamma_{S}^{h}(\mathbf{I})$ by a (flat) disk structuring element $S$ of radius 10 obtained using the look-up table algorithm detailed in [21]. Note some irregularities at the border of the head of the red parrot. Similar irregularities can be observed by considering more complex $h$-mappings such as the one discussed in [19] and [15].

\section{Approximation of $h$-Increasing Operators}

Despite the many successful applications of $h$-morphological operators, they usually are too sensitive to the $h$-ordering mapping. Furthermore, there can be many possible sources of uncertainty when dealing with an $h$-ordering which may affect the outcome of an $h$-morphological operator. For instance, under real physical conditions, there exist measurement errors when acquiring a vector-valued image. Also, the inevitable presence of rounding errors may cause some distortions on an $h$-ordering. From the methodological point of view, we may be uncertain about the method, the parameters, or the data used to determine the ordering mapping. In the light of these remarks, let us assume that we only have an approximation $\tilde{h}: \mathcal{V} \rightarrow \mathcal{L}$ of the ideal unknown ordering mapping $h: \mathcal{V} \rightarrow \mathcal{L}$. Also, let us assume that the complete lattice $\mathbb{L}$ as well as the set of lattice-valued images $\mathcal{L}$ are metric spaces.

According to the previous section, given a vector-valued image $\mathbf{I} \in \mathcal{V}$ and an increasing morphological operator $\psi: \mathcal{L} \rightarrow \mathcal{L}$, a $\tilde{h}$-morphological operator $\psi^{\tilde{h}}: \mathcal{V} \rightarrow \mathcal{V}$ yields a vector-valued image $\mathbf{J}=\psi^{\tilde{h}}(\mathbf{I}) \in \mathcal{V}$ such that

$$
\tilde{h} \mathbf{J}=\psi(\tilde{h} \mathbf{I}) .
$$

Now, if we are uncertain about the ordering mapping $\tilde{h}$, we propose to replace (10) by the inequality

$$
d_{\mathcal{L}}(\tilde{h} \mathbf{J}, \psi(\tilde{h} \mathbf{I})) \leq \tau,
$$

where $\tau \geq 0$ is a prescribed tolerance parameter and $d_{\mathcal{L}}: \mathcal{L} \times \mathcal{L} \rightarrow[0,+\infty)$ is a metric. On the one hand, $\tau=0$ implies the equality (10), which means we have complete confidence in the observed ordering function $\tilde{h}: \mathcal{V} \rightarrow \mathcal{L}$. On the other hand, we have no confidence in the ordering function when $\tau$ is sufficiently large. In this second case, the vector-valued image $\mathbf{J}$ must be determined using other criteria.

In order to prevent the occurrence of false values, we impose that the range of the output image is contained in the range of the input image. More precisely, in order to preserve local information, we impose that $\mathbf{J}(p)$ is a value in a neighborhood $B_{p} \subset \mathcal{D}$ of the pixel $p \in \mathcal{D}$. Formally, we have

$$
\mathbf{J}(p) \in \mathbf{I}\left(B_{p}\right), \quad \forall p \in \mathcal{D} .
$$

We refer to $B_{p} \subset \mathcal{D}$ as the local window. Usually, we define $B_{p}=\{p+q: q \in B\} \cap \mathcal{D}$ where, like the structuring element, $B$ is a finite subset of either $E=\mathbb{R}^{2}$ or $E=Z^{2}$.

Apart from the constraints (11) and (12), we consider an appropriate objective to be minimized such as the irregularities of the output image $\mathbf{J}$. The objective can also circumvent ambiguities when there exists more than one feasible vector-value image 
J satisfying both (11) and (12). In mathematical terms, the objective to be minimized is described by a functional $F: \mathcal{V} \rightarrow \mathbb{R}$ which associates to a vector-valued image a certain scalar measure. Concluding, we define a new morphological operator as a minimum point of the objective function $F$ subject to the constraints (11) and (12).

\section{1 $\tau$-Morphological Operator}

Given an increasing morphological operator $\psi: \mathcal{L} \rightarrow \mathcal{L}$, an uncertain ordering mapping $\tilde{h}: \mathcal{V} \rightarrow \mathcal{L}$, an objective function $F: \mathcal{V} \rightarrow \mathbb{R}$, a local window $B$, and a tolerance $\tau \geq 0$, we define the $\tau$-morphological operator $\psi^{\tau}: \mathcal{V} \rightarrow \mathcal{V}$ by setting $\psi^{\tau}(\mathbf{I})=\mathbf{J}^{*}$ where $\mathbf{J}^{*}$ is an optimal solution of the optimization problem:

$$
\begin{cases}\text { minimize } & F(\mathbf{J}) \\ \text { subject to } & d_{\mathcal{L}}(\tilde{h} \mathbf{J}, \psi(\tilde{h} \mathbf{I})) \leq \tau \\ & \mathbf{J}(p) \in \mathbf{I}\left(B_{p}\right), \quad \forall p \in \mathcal{D} .\end{cases}
$$

Note that the optimization problem (13) is feasible if $\psi(\tilde{h} \mathbf{I})(p) \in \tilde{h} \mathbf{I}\left(B_{p}\right)$ for all $p \in \mathcal{D}$. Indeed, if for any $p \in \mathcal{D}$ there exists $q \in B_{p}$ such that $\psi(\tilde{h} \mathbf{I})(p)=\tilde{h} \mathbf{I}(q)$, then setting $\mathbf{J}(p)=\mathbf{I}(q)$ yields an image $\mathbf{J}$ such that (11) holds true for any $\tau \geq 0$. For example, the optimization problem (13) is feasible if one considers a classical erosion or a classical dilation by a flat structuring element $S$ which is included in the local window $B$, that is, $S \subset B$. Apart from the feasibility issues, the optimization problem (13) can be computationally intractable. Thus, let us present a simplified version of this problem which scales linearly in the size of the image and the number of bands or channels.

A simplified problem is obtained if the objective function $F: \mathbb{V}^{\mathcal{D}} \rightarrow \mathbb{R}$ is formulated in a pointwise manner as follows where $F_{p}: \mathbb{V} \rightarrow \mathbb{R}$, for all $p \in \mathcal{D}$ :

$$
F(\mathbf{J})=\sum_{p \in \mathcal{D}} F_{p}(\mathbf{J}(p))
$$

Although $F_{p}$ may change for each pixel $p \in \mathcal{D}$, it depends only on the value $\mathbf{x}=\mathbf{J}(p)$. For example, we can define the objective function $F_{p}: \mathbb{V} \rightarrow \mathbb{R}$ as follows for all $p \in \mathcal{D}$ where $\mathbf{R} \in \mathbb{V}^{\mathcal{D}}$ is a reference vector-valued image and $d_{\mathbb{L}}$ denotes a metric on $\mathbb{L}$ :

$$
F_{p}(\mathbf{x})=d_{\mathbb{L}}(\mathbf{x}, \mathbf{R}(p)) .
$$

More importantly, by considering a pointwise ordering mapping $\tilde{h}: \mathbb{V} \rightarrow \mathcal{L}$ and the maximum metric $d_{\mathcal{L}}(\mathbf{I}, \mathbf{J})=\max \left\{d_{\mathbb{L}}(\mathbf{I}(p), \mathbf{J}(p)): p \in \mathcal{D}\right\}$, the optimization problem (13) can be decomposed into the following optimization problems for all $p \in \mathcal{D}$ :

$$
\begin{cases}\text { minimize } & F_{p}(\mathbf{x}), \\ \text { subject to } & d_{\mathbb{L}}(\tilde{h}(\mathbf{x}), \psi(\tilde{h} \mathbf{I})(p)) \leq \tau, \quad \mathbf{x} \in \mathbf{I}\left(B_{p}\right) .\end{cases}
$$

Then, the pointwise $\tau$-morphological operator $\psi^{\tau}: \mathbb{V}^{\mathcal{D}} \rightarrow \mathbb{V}^{\mathcal{D}}$ is defined by setting, for all $p \in \mathcal{D}, \psi^{\tau}(\mathbf{I})(p)=\mathbf{x}^{*}$, where $\mathbf{x}^{*}$ is an optimal solution of (16). 
In the worst case, a solution of (16) can be obtained by an exhaustive search on $\mathbf{I}\left(B_{p}\right)$. Moreover, such exhaustive search requires $\operatorname{Card}(B)$ evaluations of $F_{p}$ and $d_{\mathbb{L}}$, which are usually linear on the number of bands or channels of the multivalued image. Thus, the vector-valued image $\psi^{\tau}(\mathbf{I})$ can be obtained performing $\mathcal{O}(\operatorname{Card}(\mathcal{D}) \cdot \operatorname{Card}(B))$ operations and evaluations of the objective functions and the metric $d_{\mathbb{L}}$.

Like (13), the optimization problems given by (16) are all feasible if $\psi(\tilde{h} \mathbf{I})(p) \in$ $\tilde{h} \mathbf{I}\left(B_{p}\right), \forall p \in \mathcal{D}$. In our implementations, we alert the user and define $\psi^{\tau}(\mathbf{I})(p)=\mathbf{I}(p)$ if the optimization problem (16) is unfeasible for some $p \in \mathcal{D}$.

\section{2 $\lambda$-Morphological Operator}

Alternatively, instead of using the restriction $d_{\mathcal{L}}(\tilde{h} \mathbf{J}, \psi(\tilde{h} \mathbf{I})) \leq \tau$, we may add this term multiplied by a scaling factor $\lambda \geq 0$ in the objective function. The resulting optimization problem is:

$$
\begin{cases}\operatorname{minimize} & F(\mathbf{J})+\lambda d_{\mathcal{L}}(\tilde{h} \mathbf{J}, \psi(\tilde{h} \mathbf{I})), \\ \text { subject to } & \mathbf{J}(p) \in \mathbf{I}\left(B_{p}\right) .\end{cases}
$$

Given an increasing morphological operator $\psi: \mathcal{L} \rightarrow \mathcal{L}$, an uncertain mapping $\tilde{h}$ : $\mathcal{V} \rightarrow \mathcal{L}$, an objective function $F: \mathcal{V} \rightarrow \mathbb{R}$, a local window $B$, and a parameter $\lambda \geq 0$, we define the $\lambda$-morphological operator $\psi^{\lambda}: \mathbb{V}^{\mathcal{D}} \rightarrow \mathbb{V}^{\mathcal{D}}$ by means of the equation $\psi^{\lambda}(\mathbf{I})=\mathbf{J}^{*}$, where $\mathbf{J}^{*}$ is an optimal solution of (17) for a given $\mathbf{I} \in \mathbb{V}^{\mathcal{D}}$.

We would like to call the reader's attention to two important issues related to the $\lambda$-morphological operator $\psi^{\lambda}$ defined by means of (17). First, the parameter $\lambda \geq 0$ in (17) controls the trade-off between the objective function and the ordering mapping $\tilde{h}$. Specifically, the larger the parameter $\lambda \geq 0$ the higher the confidence in the observed mapping $\tilde{h}$. Indeed, if $\lambda$ is assigned a small value, the ordering mapping $\tilde{h}$ is considered to be uncertain and less emphasis is placed on it. At the other extreme, the similarity between $\tilde{h} \mathbf{J}$ and $\psi(\tilde{h} \mathbf{I})$ dominates the objective function when $\lambda$ is sufficiently large.

Secondly, but not less important, the optimization problem (17) is always feasible. Thus, in contrast (16), it does not require $\psi(\tilde{h} \mathbf{I})(p) \in \tilde{h} \mathbf{I}\left(B_{p}\right), \forall p \in \mathcal{D}$. As a consequence, $\psi^{\lambda}: \mathbb{V}^{\mathcal{D}} \rightarrow \mathbb{V}^{\mathcal{D}}$ is well-defined even when the underlying morphological operator $\psi: \mathbb{L}^{\mathcal{D}} \rightarrow \mathbb{L}^{\mathcal{D}}$ is based on a non-flat structuring element.

Finally, in analogy to the previous $\tau$-morphological operator, (17) can be simplified if the objective function $F: \mathbb{V}^{\mathcal{D}} \rightarrow \mathbb{R}$ can be written as (15) and the metric $d_{\mathcal{L}}$ is given by $d_{\mathcal{L}}(\mathbf{I}, \mathbf{J})=\sum_{p \in \mathcal{D}} d_{\mathbb{L}}(\mathbf{I}(p), \mathbf{J}(p))$, where $d_{\mathbb{L}}$ denotes a metric on $\mathbb{L}$. Using exhaustive search, the $\lambda$-morphological operator $\psi^{\lambda}$ requires $\mathcal{O}(\operatorname{Card}(\mathcal{D}) \cdot \operatorname{Card}(B))$ operations and evaluations of the objective functions and $d_{\mathbb{L}}$. GNU Octave sourcecodes of both $\tau$-morphological and $\lambda$-morphological operators are available at https : //codeocean.com/capsule/3486771/tree/v1.

\subsection{Computational Experiments}

This subsection provides some computational experiments to illustrate the new multivalued morphological operators. For simplicity, let us consider the color image I shown in Fig. 1a), an opening $\gamma_{S}$ by a disk $S$ of radius 10 as the increasing morphological operator $\psi$, a disk of radius 20 as the local window $B$, and the functional $F_{p}: \mathbb{V}_{\mathrm{RGB}} \rightarrow \mathbb{R}$ 


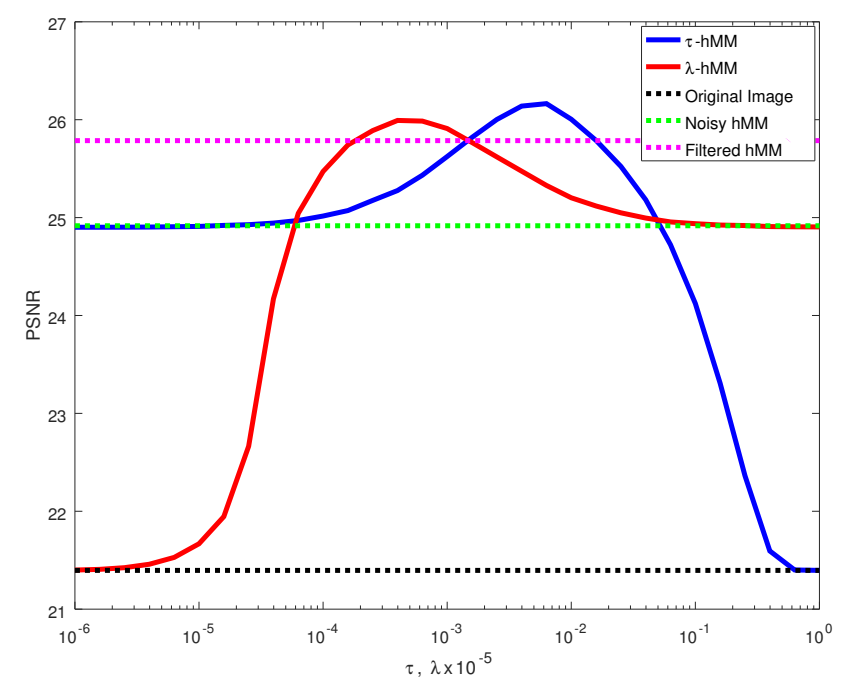

Fig. 2. PSNR between the ideal $h$-opening $\gamma_{S}^{h}(\mathbf{I})$ and the openings $\gamma_{S}^{\tau}(\mathbf{I}), \gamma_{S}^{\lambda}(\mathbf{I}), \gamma_{S}^{\tilde{h}}(\mathbf{I}), \gamma_{S}^{g \tilde{h}}(\mathbf{I})$, or the original image $\mathbf{I}$ by the parameters $\tau$ or $\lambda \times 10^{-5}$.

given by (15) with the usual Euclidean distance. Also, let us suppose we only know a noisy version $\tilde{h}: \mathbb{V}_{\mathrm{RGB}} \rightarrow[0,1]$ of the (ideal) ordering mapping $h: \mathbb{V}_{\mathrm{RGB}} \rightarrow[0,1]$ given by (4). In our experiments, $\tilde{h}$ is obtained by adding uncorrelated Gaussian noise with zero mean and variance $\sigma^{2}=0.01$, i.e., $\tilde{h}(\mathbf{x})=\max \{0, \min \{1, h(\mathbf{x})+N\}\}$, where $N \sim \mathcal{N}(0,0.01)$.

First, let us address the effect of the parameters $\tau \geq 0$ and $\lambda \geq 0$ on the operators $\gamma_{S}^{\tau}$ and $\gamma_{S}^{\lambda}$ defined respectively by (16) and (17) with $\psi=\gamma_{S}$ and the reference image $\mathbf{R}=\mathbf{I}$. Quantitatively, Fig. 2 shows the average peak signal-to-noise ratio (PSNR) between the ideal $h$-opening $\gamma_{S}^{h}(\mathbf{I})$ and the operators $\gamma^{\tau}$ and $\gamma^{\lambda}$ for different values of the parameters $\tau \geq 0$ and $\lambda \geq 0$. The average PSNR have been obtained by performing the same experiment 20 times for each value of the parameters $\tau$ and $\lambda$. For comparison purposes, we included in Fig. 2 the horizontal lines corresponding to the PSNR values between the ideal $h$-opening $\gamma_{S}^{h}(\mathbf{I})$ and the original image $\mathbf{I}$, the noisy $\tilde{h}$-opening $\gamma_{S}^{\tilde{h}}(\mathbf{I})$, and the filtered $g \tilde{h}$-opening $\gamma_{S}^{g \tilde{h}}(\mathbf{I})$. Here, $\gamma_{S}^{\tilde{h}}(\mathbf{I})$ and $\gamma_{S}^{g \tilde{h}}(\mathbf{I})$ have been computed using the look-up table algorithm with $\tilde{h} \mathbf{I}$ and $g \tilde{h} \mathbf{I}$, respectively, where $g$ denotes an isotropic Gaussian filter with spread 0.5 . In other words, the filtered $g \tilde{h}$-opening is obtained by trying to remove the Gaussian noise from $\tilde{h} \mathbf{I}$ using a Gaussian filter before computing the $h$-morphological operator. As expected, we have

$$
\lim _{\tau \rightarrow 0} \operatorname{PSNR}\left(\gamma_{S}^{\tau}(\mathbf{I}), \gamma_{S}^{h}(\mathbf{I})\right)=\operatorname{PSNR}\left(\gamma_{S}^{\tilde{h}}(\mathbf{I}), \gamma_{S}^{h}(\mathbf{I})\right)=\lim _{\lambda \rightarrow \infty} \operatorname{PSNR}\left(\gamma_{S}^{\lambda}(\mathbf{I}), \gamma_{S}^{h}(\mathbf{I})\right),
$$

and

$$
\lim _{\tau \rightarrow \infty} \operatorname{PSNR}\left(\gamma_{S}^{\tau}(\mathbf{I}), \gamma_{S}^{h}(\mathbf{I})\right)=\operatorname{PSNR}\left(\mathbf{I}, \gamma_{S}^{h}(\mathbf{I})\right)=\lim _{\lambda \rightarrow 0} \operatorname{PSNR}\left(\gamma_{S}^{\lambda}(\mathbf{I}), \gamma_{S}^{h}(\mathbf{I})\right)
$$




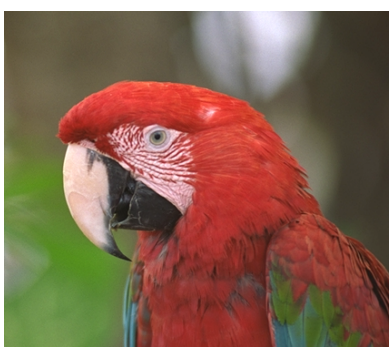

a) I

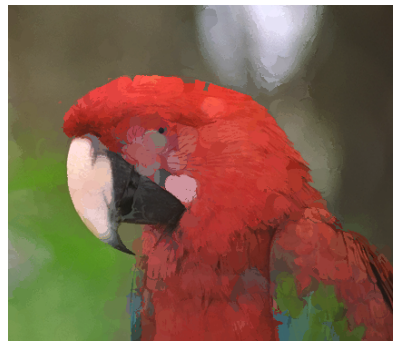

d) $\gamma_{S}^{\tau}(\mathbf{I})$

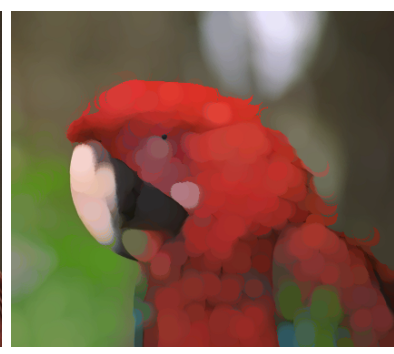

b) $\gamma_{S}^{h}(\mathbf{I})$

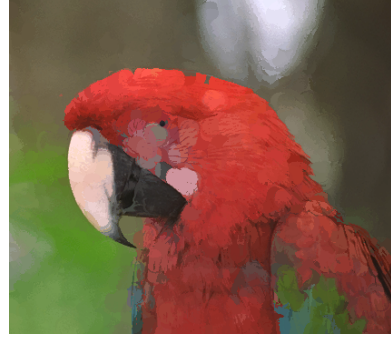

f) $\gamma_{S}^{\lambda}(\mathbf{I})$

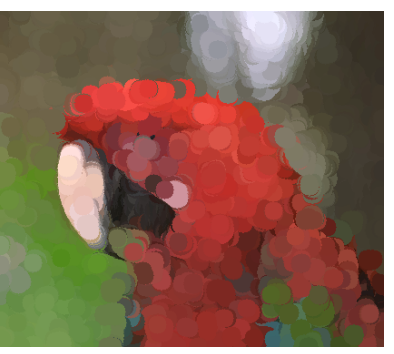

c) $\gamma_{S}^{\tilde{h}}(\mathbf{I})$

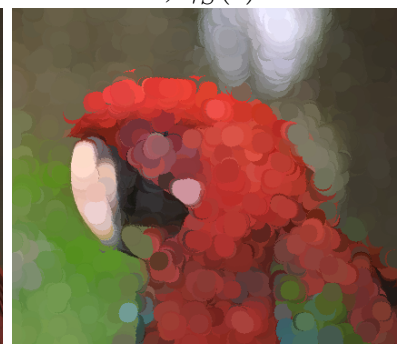

c) $\gamma_{S}^{g \tilde{h}}(\mathbf{I})$

Fig. 3. Color image $\mathbf{I}$ and its openings by a disk of radius 10 obtained using different approaches.

Most importantly, the largest PSNR rates are obtained for $\tau=0.0063$ and $\lambda=39.81$. For these values, the new operators outperformed the $g \tilde{h}$-opening obtained by filtering the noisy $\tilde{h} \mathbf{I}$ before applying the look-up table algorithm [21]. A visual interpretation of the original image $\mathbf{I}$ and the outcome of the openings $\gamma_{S}^{h}(\mathbf{I}), \gamma_{S}^{\tilde{h}}(\mathbf{I}), \gamma_{S}^{\tau}(\mathbf{I})$, $\gamma_{S}^{\lambda}(\mathbf{I})$, and $\gamma_{S}^{g \tilde{h}}(\mathbf{I})$ is shown in Fig. 3. Comparing Figs. 3b) and c), we observe how $h$-morphological operators are sensitive to the ordering mapping. We can also visualize a significant improvement on the outcome of the new operators $\gamma_{S}^{\tau}(\mathbf{I})$ and $\gamma_{S}^{\lambda}(\mathbf{I})$, including some kind of regularity in the edge of the head of the red parrot.

Finally, let us turn our attention to the objective function. We previously considered $\mathbf{R}=\mathbf{I}$ but, evidently, another image can be used as the reference image. Fig. 4 shows the images $\gamma_{S}^{\tau}(\mathbf{I})$ and $\gamma_{S}^{\lambda}(\mathbf{I})$ produced by the new operators using $\tau=0.02$ and $\lambda=60$ but considering $\mathbf{R}=\gamma_{S}^{M}(\mathbf{I})$, where $\gamma_{S}^{M}$ denotes the opening by a disk of radius 10 obtained using the marginal approach. As a consequence, $\gamma_{S}^{\tau}(\mathbf{I})$ and $\gamma_{S}^{\lambda}(\mathbf{I})$ approximate the marginal opening $\gamma_{S}^{M}$, constrained by the noisy $\tilde{h}$-mapping, but without introducing false colors. In this simple example, the openings shown in Fig. 4 circumvented the irregularity issues present in the openings shown in Figs. 1 and 3. Furthermore, it reveals some of the potential advantages of the new operators $\psi^{\tau}$ and $\psi^{\lambda}$.

\section{Concluding Remarks}

Despite the rich mathematical background and its successful applications for analysis and processing of color and hyperspectral images [9, 20,21], morphological operators 


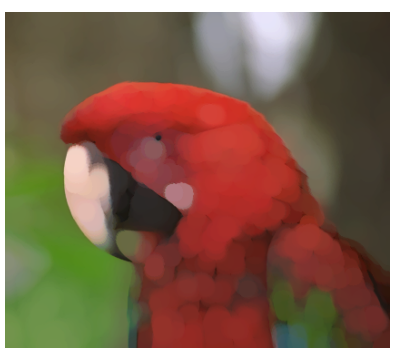

(a) $\gamma_{S}^{\mathrm{M}}(\mathbf{I})$

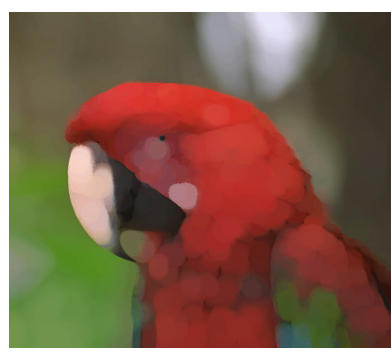

(b) $\gamma_{S}^{\tau}(\mathbf{I})$

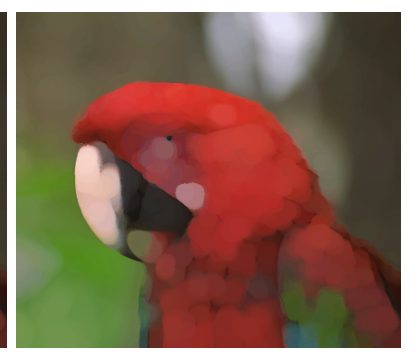

(c) $\gamma_{S}^{\lambda}(\mathbf{I})$

Fig. 4. Marginal opening $\gamma_{S}^{M}$ by a disk $S$ of radius 10 and the corresponding $\gamma_{S}^{\tau}$ and $\gamma_{S}^{\lambda}$ operators using $\gamma_{S}^{M}$ as the reference in (15) and the parameters $\tau=2 \cdot 10^{-2}$ and $\lambda=60$.

based on reduced orderings are usually too sensitive to the ordering mapping. In many practical situations, however, the ordering mapping $h$ is subject to uncertainties such as the unavoidable measurement errors.

In this paper, by assuming that the ordering mapping is uncertain, we proposed two approaches which are formulated as the solution of optimization problems. Precisely, we assume that we only have an approximation $\tilde{h}$ of the ideal ordering mapping $h$. Given an increasing morphological operator $\psi: \mathcal{L} \rightarrow \mathcal{L}$, we define the $\tau$-operator $\psi^{\tau}$ and the $\lambda$-operator $\psi^{\lambda}$ as the solution of the optimization problems (13) and (17), respectively. Apart from the capability to deal with an uncertain ordering mapping, the new operators can circumvent both the false values problem and the irregularity issues found in many approaches to multivalued mathematical morphology $[6,17]$. In fact, we avoid false values by imposing $\mathbf{J}(p) \in \mathbf{I}\left(B_{p}\right)$, where $B_{p}$ is a local window. The irregularity issue can be dealt by considering an adequate objective function. Finally, both optimization problems (13) and (17) scales linearly in the size of the image and the local window if the objective function can be decomposed in a pointwise manner according to (15).

In order to illustrate the new operators and how they are able to deal with the uncertainty present in the ordering mapping, we presented some simple computational experiments with a color image in the RGB color space. By considering an uncertain ordering mapping $\tilde{h}$ obtained by adding Gaussian noise, both $\tau$-opening and $\lambda$-opening showed to get closer to the ideal $h$-opening than the usual approach with or without a filter. The proposed approaches also significantly reduced the visual irregularity present in the images. To illustrate the effects of the choice of the objective function, we used the proposed operators to approximate the marginal opening, which is fairly regular. Visually, both $\tau$-opening and $\lambda$-opening are very similar to the marginal opening but they do not introduce false colors.

Future work relating to this paper includes studying how the new operators, in particular the $\lambda$-operators given by (17), work with non-flat structuring elements. One can also investigate and compare the performance of the new operators in applications such as image classification or segmentation. 


\section{References}

1. Angulo, J.: Morphological colour operators in totally ordered lattices based on distances: Application to image filtering, enhancement and analysis. Computer Vision and Image Understanding 107(1-2), 56-73 (July-August 2007), special issue on color image processing

2. Aptoula, E., Lefèvre, S.: A Comparative Study on Multivariate Mathematical Morphology. Pattern Recognition 40(11), 2914-2929 (November 2007)

3. Birkhoff, G.: Lattice Theory. American Mathematical Society, Providence, 3 edn. (1993)

4. Braga-Neto, U., Goutsias, J.: Supremal multiscale signal analysis. SIAM Journal of Mathematical Analysis 36(1), 94-120 (2004)

5. Chanussot, J., Lambert, P.: Total ordering based on space filling curves for multivalued morphology. In: Proceedings of the Fourth International Symposium on Mathematical Morphology and Its Applications to Image and Signal Processing. pp. 51-58. ISMM '98, Kluwer Academic Publishers, Norwell, MA, USA (1998)

6. Chevallier, E., Angulo, J.: The Irregularity Issue of Total Orders on Metric Spaces and Its Consequences for Mathematical Morphology. Journal of Mathematical Imaging and Vision 54(3), 344-357 (Mar 2016)

7. Comer, M.L., Delp, E.J.: Morphological operations for color image processing. Journal of Electronic Imaging 8(3), 279-289 (1999)

8. Gonzalez-Hidalgo, M., Massanet, S., Mir, A., Ruiz-Aguilera, D.: On the Choice of the Pair Conjunction-Implication Into the Fuzzy Morphological Edge Detector. IEEE Transactions on Fuzzy Systems 23(4), 872-884 (Aug 2015)

9. Goutsias, J., Heijmans, H.J.A.M., Sivakumar, K.: Morphological Operators for Image Sequences. Computer vision and image understanding 62, 326-346 (1995)

10. Grätzer, G., et al.: General Lattice Theory. Birkhäuser Verlag, Basel, Switzerland, 2nd edn. (2003)

11. van de Gronde, J., Roerdink, J.: Group-Invariant Colour Morphology Based on Frames. IEEE Transactions on Image Processing 23(3), 1276-1288 (March 2014)

12. Heijmans, H.J.A.M.: Mathematical Morphology: A Modern Approach in Image Processing Based on Algebra and Geometry. SIAM Review 37(1), 1-36 (1995)

13. Lézoray, O.: Complete lattice learning for multivariate mathematical morphology. Journal of Visual Communication and Image Representation 35, 220-235 (Feb 2016)

14. Ronse, C.: Why Mathematical Morphology Needs Complete Lattices. Signal Processing 21(2), 129-154 (1990)

15. Sangalli, M., Valle, M.E.: Color mathematical morphology using a fuzzy color-based supervised ordering. In: North American Fuzzy Information Processing Society Annual Conference. pp. 278-289. Springer (2018)

16. Serra, J.: A Lattice Approach to Image Segmentation. Journal of Mathematical Imaging and Vision 24, 83-130 (2006)

17. Serra, J.: The "False Colour" Problem. In: Wilkinson, M.H., Roerdink, J.B. (eds.) Mathematical Morphology and Its Application to Signal and Image Processing, Lecture Notes in Computer Science, vol. 5720, pp. 13-23. Springer Berlin Heidelberg (2009)

18. Soille, P.: Morphological Image Analysis. Springer Verlag, Berlin (1999)

19. Velasco-Forero, S., Angulo, J.: Supervised ordering in $\mathbb{R}^{p}$ : Application to morphological processing of hyperspectral images. IEEE Transactions on Image Processing 20(11), 3301$3308(2011)$

20. Velasco-Forero, S., Angulo, J.: Random projection depth for multivariate mathematical morphology. IEEE Journal of Selected Topics in Signal Processing 6(7), 753-763 (2012)

21. Velasco-Forero, S., Angulo, J.: Vector ordering and multispectral morphological image processing. In: Advances in Low-Level Color Image Processing, pp. 223-239. Springer (2014) 